Pacific Journal of Mathematic 


\title{
ON THE NORMAL SUBGROUPS OF INTEGRAL ORTHOGONAL GROUPS
}

\author{
D. G. JAMES
}

\begin{abstract}
Let $\mathscr{E}$ denote the spinorial kernel of an orthogonal group of an indefinite unimodular quadratic form over the integers in a global field. The normal subgroups of $\mathscr{E}$ that arise from the local structure of $\mathscr{E}$ are studied.
\end{abstract}

Let $S$ be a Dedekind set of spots on a global field $F$ with characteristic not two and 0 the ring of integers of $F$ at $S$. Let $V$ be a finite dimensional quadratic space over $F$ with dimension at least 5 and associated bilinear form $B$ and quadratic form $q$. The orthogonal group of $V$ is

$$
O(V)=\{\varphi \in \operatorname{End} V \mid q(\varphi(x))=q(x) \text { for all } x \in V\} .
$$

Assume $V$ supports a unimodular lattice $M$ with orthogonal group $O(M)=\{\varphi \in O(V) \mid \varphi(M)=M\}$. At each spot $\mathfrak{p} \in S$ we can localize and consider the orthogonal group $O\left(M_{p}\right)$ of the isotropic unimodular lattice $M_{\mathfrak{p}}$ over the ring of integers $\mathfrak{o}_{\mathfrak{p}}$ in the local field $F_{\mathfrak{p}}$. The subgroups of $O\left(M_{\mathfrak{p}}\right)$ normalized by its commutator subgroup have been classified in $[1,2,3]$. We show here how this local structure can be injected into $O(M)$ when $S$ is an indefinite set of spots for $V$. A rich structure of normal subgroups of the spinorial kernel of $O(M)$ is then provided by the local behaviour at the dyadic spots. Most of our terminology and notation is taken from O'Meara [4].

1. $\mathscr{E}$-invariant sublattices of $M, M_{\mathfrak{p}}$ is split by a hyperbolic plane $H_{\mathfrak{p}}$ at each spot $\mathfrak{p} \in S$. Write $M_{\mathfrak{p}}=H_{\mathfrak{p}} \perp K_{\mathfrak{p}}$ and $H_{\mathfrak{p}}=\mathfrak{D}_{\mathfrak{p}} u_{\mathfrak{p}}+$ $\mathfrak{o}_{\mathfrak{p}} v_{\mathfrak{p}}$ where $B_{\mathfrak{p}}\left(u_{\mathfrak{p}}, v_{\mathfrak{p}}\right)=1$ and $q_{\mathfrak{p}}\left(u_{\mathfrak{p}}\right)=q_{\mathfrak{p}}\left(v_{\mathfrak{p}}\right)=0$. In [2] it is shown that $\mathscr{E}_{p}$, the group generated by the Siegel transformations $E\left(u_{\mathfrak{p}}, x_{\mathfrak{p}}\right)$ and $E\left(v_{p}, x_{p}\right)$, is equal to the spinorial kernel of $O\left(M_{p}\right)$. Define

$$
\mathscr{E}=\left\{\varphi \in O(M) \mid \varphi_{p} \in \mathscr{E}_{p} \text { for all } \mathfrak{p} \in S\right\} .
$$

Note that $\mathscr{E}$ is defined if $\operatorname{dim} V \geqq 5$, even when $V$ is anisotropic. In fact, using the global square theorem [4, 65:15] and [2, Theorem 2.9], it is easily seen that $\mathscr{E}$ is the spinorial kernel of $O(M)$.

A sublattice $P$ of $M$ is called $\mathscr{E}$-invariant if $\varphi(P)=P$ for all $\varphi \in \mathscr{E}$. Let $M_{*}$ denote the lattice

$$
M_{*}=\{x \in M \mid q(x) \in \mathfrak{p}\}
$$

with dual lattice 


$$
M^{*}=\left\{x \in V \mid B\left(x, M_{*}\right) \subseteq \mathfrak{o}\right\} .
$$

Define an ideal $\mathfrak{a}(P)$ in $\mathfrak{D}$ by

$$
a(P)=\sum_{r \in P} B\left(r, M_{*}\right) .
$$

Then

$$
a(P)^{-1} P \subseteq M^{*}
$$

Lemma 1.1. Let $P$ be sublattice of $M$ and

$$
M_{*} \leqq \mathfrak{a}(P)^{-1} P \subseteq M^{*} .
$$

Then $P$ is $\mathscr{E}$-invariant.

Proof. Locally, $P_{\mathfrak{p}}$ is $\mathscr{C}_{\mathfrak{p}}$-invariant [2, Theorem 3.1] since $\left(M_{*}\right)_{\mathfrak{p}}=$ $\left(M_{\mathfrak{p}}\right)_{*}, \mathfrak{a}\left(P_{\mathfrak{p}}\right)=\mathfrak{a}(P)_{\mathfrak{p}}$ and

$$
\left(M_{\mathfrak{p}}\right)_{*} \subseteq \mathfrak{a}\left(P_{\mathfrak{p}}\right)^{-1} P_{\mathfrak{p}} \subseteq\left(M_{\mathfrak{p}}\right)^{*} .
$$

Take $\varphi \in \mathscr{E}$ and let $\varphi(P)=Q$. Then $\varphi_{p} \in \mathscr{E}_{p}$ and

$$
P_{\mathrm{p}}=\varphi_{\mathrm{p}}\left(P_{\mathrm{p}}\right)=\varphi(P)_{\mathrm{p}}=Q_{\mathrm{p}}
$$

for all $\mathfrak{p} \in S$. Hence $P=Q$ by $[4,81 \mathrm{E}]$.

THEOREM 1.2. Let $S$ be an indefinite set of spots for $V$ and assume $\operatorname{dim} V \geqq 7$ if $\left[\mathfrak{o}_{\mathfrak{p}}: \mathfrak{p}\right]=2$ at any dyadic spot. Then a sublattice $P$ of $M$ is $\mathscr{E}$-invariant if and only if

$$
M_{*} \subseteq \mathfrak{a}(P)^{-1} P \subseteq M^{*} \text {. }
$$

Proof. We need only show that if $P$ is $\mathscr{E}$-invariant, then $\left(M_{\mathfrak{p}}\right)_{*} \subseteq$ $\mathfrak{a}\left(P_{\mathfrak{p}}\right)^{-1} P_{\mathfrak{p}}$ for all $\mathfrak{p} \in S$. Fix $\mathfrak{p} \in S$ and take $\theta_{\mathfrak{p}} \in \mathscr{E}_{\mathfrak{p}}$. By the strong approximation theorem for rotations [4, 104: 4], there exists $\varphi \in O(V)$ such that $\left\|\varphi-\theta_{\mathfrak{p}}\right\|_{\mathfrak{p}}<\varepsilon$ and $\|\varphi\|_{\mathfrak{q}}=1$ for $\mathfrak{q} \neq \mathfrak{p}$. Then $\varphi \in O(M)$ and, since $\mathscr{E}$ is the spinorial kernel, $\varphi \in \mathscr{E}$. Hence $\varphi(P)=P$. By making $\varepsilon$ sufficiently small, we may assume $\left(\varphi_{\mathfrak{p}}-\theta_{\mathfrak{p}}\right)\left(P_{\mathfrak{p}}\right) \cong P_{\mathfrak{p}}$. Thus $\theta_{\mathfrak{p}}\left(P_{\mathfrak{p}}\right)=P_{\mathfrak{p}}$ for all $\theta_{\mathfrak{p}} \in \mathscr{E}_{\mathfrak{p}}$. Hence $P_{\mathfrak{p}}$ is $\mathscr{E}_{p}$-invariant and by [2, Theorem 3.1] it follows that $a\left(P_{p}\right)\left(M_{p}\right)_{*} \subseteq P_{p}$.

THEOREM 1.3. For each dyadic spot $\mathfrak{p}$ assume given an $\mathfrak{b}_{p}$-lattice $J_{p}$ with $\left(M_{\mathfrak{p}}\right)_{*} \subseteq J_{\mathfrak{p}} \subseteq\left(M_{\mathfrak{p}}\right)^{*}$. Then there exists an o-lattice $P$ such that $P_{\mathfrak{p}}=J_{\mathfrak{p}}$ at each dyadic spot and $M_{*} \subseteq P \subseteq M^{*}$.

Proof. This follows immediately from [4, 81:14] since $\left(M_{\mathfrak{p}}\right)_{*}=$ $\left(M_{\mathfrak{p}}\right)^{*}=M_{\mathfrak{p}}$ at all nondyadic spots. 
REMARK 1.4. If 2 is unramified, the lattices satisfying $\left(M_{2}\right)_{*} \subseteq$ $P \subseteq\left(M_{2}\right)^{*}$ were determined in [1]. When 2 ramifies the number of such lattices proliferates (even for the gaussian integers, there are lattices $M$ having more than 20 such $P$ ).

2. Normal subgroups of $\mathscr{E}$. Let $\Xi$ be an indexing set such that $M_{\xi}, \xi \in \Xi$, gives all the lattices on $V$ such that

$$
M_{*} \subseteq M_{\xi} \subseteq M^{*}
$$

Let $\mathfrak{a}$ be an ideal in $\mathfrak{D}$ such that $a M_{\xi} \subseteq M_{*}$. Then $a_{\mathfrak{p}} M_{\xi \mathfrak{p}} \subseteq\left(M_{\mathfrak{p}}\right)_{*}$ and we define $\mathscr{E}_{p}\left(a_{p} M_{\xi p}\right)$ as in [2] as the normal subgroup of $\mathscr{E}_{p}$ generated by all isometries of the form $\theta_{\mathfrak{p}} E\left(u_{\mathfrak{p}}, x_{\mathfrak{p}}\right) \theta_{\mathfrak{p}}^{-1}$ or $\theta_{\mathfrak{p}} E\left(v_{\mathfrak{p}}, x_{\mathfrak{p}}\right) \theta_{\mathfrak{p}}^{-1}$ where $\theta_{p} \in \mathscr{E}_{p}$ and $x_{\mathfrak{p}} \in K_{\mathfrak{p}} \cap \mathfrak{a}_{\mathfrak{p}} M_{\hat{\xi} p}$. Define

$$
\mathscr{E}\left(\mathfrak{a} M_{\xi}\right)=\left\{\varphi \in \mathscr{E} \mid \varphi_{p} \in \mathscr{E}_{p}\left(a_{\mathfrak{p}} M_{\hat{\xi} p}\right) \text { for all } p \in S\right\}
$$

and

$$
\mathscr{F}\left(a M_{\xi}\right)=\left\{\varphi \in \mathscr{E} \mid[\varphi, \mathscr{E}] \subseteq \mathscr{E}\left(a M_{\xi}\right)\right\} .
$$

Then $\mathscr{E}\left(a M_{\xi}\right) \subseteq \mathscr{F}\left(a M_{\xi}\right)$ and any subgroup $\mathscr{N}$ of $\mathscr{E}$ such that

$$
\mathscr{E}\left(a M_{\xi}\right) \subseteq \mathscr{N} \subseteq \mathscr{F}\left(a M_{\xi}\right)
$$

is a normal subgroup of $\mathscr{E}$.

We also define the local group $\mathscr{F}_{p}\left(a_{\mathfrak{p}} M_{\xi_{\mathfrak{p}}}\right)$ by

$$
\mathscr{F}_{p}\left(a_{p} M_{\xi_{p}}\right)=\left\{\varphi_{p} \in \mathscr{E}_{p} \mid\left[\varphi_{p}, \mathscr{E}_{p}\right] \subseteq \mathscr{E}_{p}\left(a_{p} M_{\xi_{p}}\right)\right\} .
$$

Note that this definition is more restrictive than that in [2] where $\varphi_{\mathfrak{p}}$ was taken in $O\left(M_{\mathfrak{p}}\right)$, not $\mathscr{E}_{p}$.

THEOREM 2.1. Let $S$ be an indefinite set of spots for $V$ and $\operatorname{dim} V \geqq 5$. Then for any ideal $\mathfrak{a} \neq\{0\}$ with $\mathfrak{a} M_{\xi} \subseteq M_{*}$,

$$
\mathscr{F}\left(a M_{\xi}\right) / \mathscr{E}\left(a M_{\xi}\right) \cong \prod_{p \mid a} \mathscr{F}_{p}\left(a_{p} M_{\xi_{p}}\right) / \mathscr{E}_{p}\left(a_{p} M_{\xi p}\right) \text {. }
$$

Proof. Observe first that for $\mathfrak{p} \nmid \mathfrak{a}$ we have

$$
\mathscr{E}_{p}\left(a_{p} M_{\xi p}\right)=\mathscr{E}_{p}=\mathscr{F}_{p}\left(a_{p} M_{\xi_{p}}\right) \text {. }
$$

Define a mapping

$$
\Gamma: \mathscr{F}\left(\mathfrak{a} M_{\xi}\right) \longrightarrow \prod_{\mathfrak{p} \mid a} \mathscr{F}_{p}\left(\mathfrak{a}_{\mathfrak{p}} M_{\xi_{\mathfrak{p}}}\right) / \mathscr{E}_{\mathfrak{p}}\left(\mathfrak{a}_{\mathfrak{p}} M_{\hat{\xi}_{\mathfrak{p}}}\right)
$$

by sending $\varphi \in \mathscr{F}\left(a M_{\xi}\right)$ into $\left(\cdots, \widetilde{\varphi}_{p}, \cdots\right)_{p \mid a}$ where $\widetilde{\varphi}_{p}$ denotes the coset determined by $\mathscr{\varphi}_{\mathfrak{p}}$ in $\mathscr{F}_{p}\left(a_{\mathfrak{p}} M_{\xi \mathfrak{p}}\right) / \mathscr{C}_{\mathfrak{p}}\left(a_{\mathfrak{p}} M_{\xi_{p}}\right)$. It is necessary to show that $\Gamma$ is well-defined, namely $\varphi_{\mathfrak{p}} \in \mathscr{F}_{\mathfrak{p}}\left(\mathfrak{a}_{\mathfrak{p}} M_{\xi \mathfrak{p}}\right)$ for each $\mathfrak{p} \mid \mathfrak{a}$. Let 
$\psi_{p} \in \mathscr{E}_{p}$ and take $\theta \in \mathscr{E}$ such that $\left\|\psi_{p}^{-1} \theta-I\right\|_{p}<\varepsilon$. Then $[\varphi, \theta]$ is in $\mathscr{E}\left(\mathfrak{a} M_{\xi}\right)$ and hence $\left[\varphi_{\mathfrak{p}}, \theta_{\mathfrak{p}}\right]$ is in $\mathscr{C}_{\mathfrak{p}}\left(\mathfrak{a}_{\mathfrak{p}} M_{\xi \mathfrak{p}}\right)$. For sufficiently small $\varepsilon$, it follows from Corollary 3.3 that $\psi_{\mathfrak{p}}^{-1} \theta_{\mathfrak{p}}$ is in $\mathscr{E}_{\mathfrak{p}}\left(a_{\mathfrak{p}} M_{\xi \mathfrak{p}}\right)$ and hence $\left[\varphi_{p}, \psi_{p}\right] \in \mathscr{E}_{p}\left(a_{p} M_{\xi p}\right)$. Thus $\Gamma$ is a well-defined group homomorphism. $\phi$ is in the kernel of $\Gamma$ if and only if $\phi_{\mathfrak{p}} \in \mathscr{E}_{\mathfrak{p}}\left(\mathfrak{a}_{\mathfrak{p}} M_{\xi \mathfrak{p}}\right)$ for each $\mathfrak{p} \mid$ a. But $\varphi_{\mathfrak{p}} \in \mathscr{C}_{\mathfrak{p}}=\mathscr{E}_{\mathfrak{p}}\left(\mathfrak{a}_{\mathfrak{p}} M_{\xi \mathfrak{p}}\right)$ for $\mathfrak{p} \nmid \mathfrak{a}$. Hence the kernel of $\Gamma$ is $\mathscr{E}\left(a M_{\xi}\right)$.

It remains to show that $\Gamma$ is surjective. For each $\mathfrak{p} \mid a$ fix $\psi_{\mathfrak{p}}$ in $\mathscr{F}_{p}\left(\mathfrak{a}_{\mathfrak{p}} M_{\xi \mathfrak{p}}\right)$. By the strong approximation theorem, there exists $\varphi \in \mathscr{E}$ such that

$$
\left\|\psi_{p}^{-1} \varphi-I\right\|_{\mathfrak{p}}<\varepsilon \text { for } \mathfrak{p} \mid \mathfrak{a} .
$$

It now suffices to show $\varphi \in \mathscr{F}\left(a M_{\xi}\right)$, for if $\varepsilon$ is sufficiently small, $\psi_{p}^{-1} \mathscr{\varphi}_{\mathfrak{p}} \in \mathscr{E}_{\mathfrak{p}}\left(a_{\mathfrak{p}} M_{\xi \mathfrak{p}}\right)$ by Corollary 3.3 and hence $\psi_{\mathfrak{p}}$ and $\varphi_{\mathfrak{p}}$ determine the same coset in $\mathscr{F}_{p}\left(a_{p} M_{\xi \mathfrak{p}}\right) / \mathscr{E}_{\mathfrak{p}}\left(a_{\mathfrak{p}} M_{\xi \mathfrak{p}}\right)$. We must show $\left[\mathscr{P}_{\mathfrak{p}}, \theta_{\mathfrak{p}}\right] \in$ $\mathscr{E}_{\mathfrak{p}}\left(\mathfrak{a}_{\mathfrak{p}} M_{\xi \mathfrak{p}}\right)$ for any $\theta \in \mathscr{E}$ and all $\mathfrak{p} \in S$. For $\mathfrak{p} \nmid \mathfrak{a}$ this is trivial. For $\mathfrak{p} \mid a$ and $\varepsilon$ sufficiently small, $\chi_{p}=\psi_{p}^{-1} \varphi_{p} \in \mathscr{E}_{p}\left(a_{p} M_{\xi \mathfrak{p}}\right)$ and hence

$$
\left[\varphi_{p}, \theta_{p}\right]=\left[\psi_{p} \chi_{p}, \theta_{p}\right]=\left[\psi_{p}, \chi_{p} \theta_{p} \chi_{p}^{-1}\right]\left[\chi_{p}, \theta_{p}\right]
$$

is in $\mathscr{E}_{\mathfrak{p}}\left(\mathfrak{a}_{\mathfrak{p}} M_{\xi \mathfrak{p}}\right)$. This completes the proof.

REMARK 2.2. We restricted ourselves to Dedekind domains coming from global fields so that the strong approximation theorem for rotations could be used. If, however, we assume $M$ has hyperbolic rank at least one, so that globally $M=H \perp K$ with $H=\mathfrak{v} u+\mathfrak{o} v$ a hyperbolic plane and $K$ is free, a strong approximation theorem can still be established for any Dedekind domain. Define all $\mathscr{E}_{p}$ with respect to the localization of $H$. Then, from [2, Theorem 2.9], any $\theta_{\mathfrak{p}}$ in the spinorial kernel is of the form

$$
\theta_{\mathfrak{p}}=\prod_{i=1}^{n(\mathfrak{p})} E\left(u, x_{i}(\mathfrak{p})\right) E\left(v, y_{i}(\mathfrak{p})\right)
$$

with $x_{i}(\mathfrak{p})$ and $y_{i}(\mathfrak{p})$ in $\left(K_{\mathfrak{p}}\right)_{*}$. By approximating to the coefficients of $x_{i}(\mathfrak{p})$ and $y_{i}(\mathfrak{p})$ in the Dedekind domain $\mathfrak{o}$, we can approximate to $\theta_{\mathfrak{p}}$ by an isometry in $O(M)$ for a finite number of $\mathfrak{p}$.

3. The structure of $\mathscr{F}_{p}\left(a_{\mathfrak{p}} M_{\hat{\xi}}\right) / \mathscr{E}_{\mathfrak{p}}\left(a_{\mathfrak{p}} M_{\xi \mathfrak{p}}\right)$. We close with some comments on the structure of the abelian group $\mathscr{F}_{p}\left(\mathfrak{a}_{p} M_{\xi \mathfrak{p}}\right) / \mathscr{E}_{\mathfrak{p}}\left(\mathfrak{a}_{\mathfrak{p}} M_{\xi \mathfrak{p}}\right)$. For simplicity, since only the local situation is considered in this section, the suffix $\mathfrak{p}$ is dropped and we write $\mathfrak{o}$ for $\mathfrak{o}_{\mathfrak{p}}, M$ for $M_{\mathfrak{p}}, V$ for $V_{\mathfrak{p}}$, and so on.

For $\xi \in \Xi$, let

$$
M^{\xi}=\left\{x \in V \mid B\left(x, M_{\xi}\right) \subseteq \mathfrak{p}\right\}
$$


be the dual lattice of $M_{\xi}$. Write $M_{*}=H \perp K_{*}$ and $M_{\xi}=H \perp K_{\xi}$. Then $K_{*} \subseteq K_{\xi}$ and lwe can choose a basis $x_{1}, \cdots, x_{n}$ for $K_{*}$ such that

$$
K_{*}=\mathfrak{o} x_{1}+\cdots+\mathfrak{o} x_{n}
$$

and

$$
K_{\xi}=\mathfrak{p}^{-k_{1}} x_{1}+\cdots+\mathfrak{p}^{-k_{n}} x_{n}
$$

with the integers $k_{i}$ invariants for $K_{*}, K_{\xi}$ and

$$
0 \leqq k_{1} \leqq k_{2} \leqq \cdots \leqq k_{n} .
$$

Let $y_{1}, \cdots, y_{n}$ be the dual basis of $x_{1}, \cdots, x_{n}$. Then

$$
K^{*}=\mathfrak{o} y_{1}+\cdots+\mathfrak{o} y_{n}
$$

and

$$
K^{\xi}=\mathfrak{p}^{k_{1}} y_{1}+\cdots+\mathfrak{p}^{k_{n}} y_{n} .
$$

For each $1 \leqq i \leqq n$, define

$$
\begin{aligned}
K_{\xi(i)} & =K_{*}+\mathfrak{p}^{k_{i}} K_{\xi} \\
& =\mathfrak{o} x_{1}+\cdots+\mathfrak{o} x_{i}+\mathfrak{p}^{k_{i}-k_{i+1}} x_{i+1}+\cdots+\mathfrak{p}^{k_{i}-k_{n}} x_{n}
\end{aligned}
$$

and let $K^{\xi(i)}, 1 \leqq i \leqq n$, be the dual lattices. Write $M_{\xi(i)}=H \perp K_{\xi(i)}$ and $M^{\xi(i)}=H \perp K^{\xi(i)}$. Then

$$
M_{\xi} \supseteqq M_{\xi(1)} \supseteqq M_{\xi(2)} \supseteqq \cdots \supseteqq M_{\xi(n)}=M_{*}
$$

and

$$
M^{\xi} \sqsubseteq M^{\xi(1)} \subseteq M^{\xi(2)} \cong \cdots \sqsubseteq M^{\xi(n)}=M^{*}
$$

Define congruence subgroups by

$$
\begin{aligned}
O\left(a M_{\xi}\right) & =\left\{\varphi \in \mathscr{E} \mid \varphi(r) \equiv r \bmod a M_{\xi} \text { for all } r \in M_{*}\right\}, \\
O\left(a M_{\xi}\right)^{i} & =\left\{\varphi \in O\left(a M_{\xi}\right) \mid \varphi(r) \equiv r \bmod 2 a \mathfrak{p}^{-k_{i}} M_{\xi(i)} \text { for all } r \in 2 M^{\xi(i)}\right\}
\end{aligned}
$$

for $1 \leqq i \leqq n$, and

$$
O\left(\mathfrak{a} M_{\xi}\right)^{\sharp}=\bigcap_{i=1}^{n} O\left(\mathfrak{a} M_{\xi}\right)^{i} .
$$

Since $\mathfrak{p}^{k i} M_{\xi} \subseteq M_{\xi(i)}, 1 \leqq i \leqq n$, it can be checked that

$$
\mathscr{E}\left(a M_{\xi}\right) \subseteq O\left(\mathfrak{a} M_{\xi}\right)^{\#} \subseteq O\left(\mathfrak{a} M_{\xi}\right) \subseteq \mathscr{F}\left(\mathfrak{a} M_{\xi}\right)
$$

provided that $\mathfrak{a} q\left(M_{\xi}\right) \subseteq \mathrm{o}$. 
THEOREM 3.1. For $\mathfrak{a} \subseteq 4 \mathfrak{p}$, the subgroup $O\left(\mathfrak{a} M_{\xi}\right)^{*}$ is generated by $\mathscr{E}\left(\mathfrak{a} M_{\xi}\right)$ and the isometries $\Phi(\varepsilon)$ with $\varepsilon \equiv 1 \bmod \mathfrak{a}$.

Proof. The isometries $\Phi(\varepsilon)$ are defined in [2]. Let $\varphi \in O\left(\mathfrak{a} M_{\xi}\right)^{*}$; changing $\varphi$ by the given isometries, we will reduce it to the identity mapping. Assume for some $m \leqq n$ that $\varphi\left(y_{j}\right)=y_{j}, 1 \leqq j \leqq m-1$, (consider $\varphi \in O(V)$ ). Let

$$
\varphi\left(u+y_{m}\right)=\varepsilon u+\beta v+y_{m}+\sum_{i=1}^{n} \alpha_{i} x_{i} .
$$

Since $\varphi\left(y_{j}\right)=y_{j}$, it follows that $\alpha_{j}=0$ for $1 \leqq j \leqq m-1$. For $j \geqq$ $m, u+y_{m} \in M^{\xi(j)}$ and hence $\alpha_{j} x_{j} \in \mathfrak{a p}^{-k_{j}} M_{\xi(j)}$. Thus $\alpha_{j} \in \mathfrak{a p}^{-k_{j}}$, so that $\alpha_{j} x_{j} \in \mathfrak{a} M_{\xi}, m \leqq j \leqq n$, and hence $s=\sum_{i=1}^{n} \alpha_{i} x_{i}$ is in $a M_{\xi}$. Now let

$$
\psi=E\left(u,-x_{m}\right) E\left(u,(1-\varepsilon) x_{m}\right) E\left(v, \varepsilon^{-1} s\right) \varphi E\left(u, x_{m}\right) .
$$

If $\psi$ is generated by the given isometries, so is $\phi$ (since $\varepsilon-1 \epsilon$ $\left.a \mathfrak{p}^{-k_{m}}\right)$. Since now $\psi\left(y_{j}\right)=y_{j}$ for $1 \leqq j \leqq m$, the proof is concluded by induction.

REMARK 3.2. The assumption $\mathfrak{a} \subseteq 4 \mathfrak{p}$ in the theorem is used to ensure $\mathfrak{a p}^{-k_{i}} \subseteq \mathfrak{p}$ (in particular $\varepsilon$ is now a unit). When $4 \mathfrak{o} \subseteq \mathfrak{a}$, more care is needed (see [3, Tables I, II]).

\section{COROLLARY 3.3. $O\left(4 a M_{\xi}\right)^{\#} \sqsubseteq \mathscr{E}\left(a M_{\xi}\right)$ when $a \sqsubseteq \mathfrak{p}$.}

Proof. We need only show $\Phi(\varepsilon) \in \mathscr{E}\left(a M_{\xi}\right)$ when $\varepsilon \equiv 1 \bmod 4 \mathrm{a}$. By Hensel's lemma $\varepsilon=\eta^{2}$ with $\eta \equiv 1 \bmod a$. Now $\Phi\left(\eta^{2}\right)$ is in $\mathscr{E}\left(a M_{*}\right)$ by [2, Proposition 1.2].

Corollary 3.4. $O\left(\mathfrak{a} M_{\xi}\right)=\mathscr{E}\left(\mathfrak{a} M_{\xi}\right)$ when $\mathfrak{p}$ is nondyadic.

Proof. For nondyadic $\mathfrak{p}, M_{\xi}=M=M^{*}=M_{*}$. Clearly $O(\mathfrak{o} M)=$ $\mathscr{E}$ by the definition of $O(a M)$. If $a \leqq \mathfrak{p}$, then $\varepsilon \equiv 1 \bmod \mathfrak{a}$ and by Hensel's lemma $\varepsilon=\eta^{2}$ with $\eta \equiv 1 \bmod a$. Again, $\Phi(\varepsilon)$ is in $\mathscr{E}(a M)$.

For dyadic $\mathfrak{p}$, under the hypothesis of Theorem $3.1, O\left(a M_{\xi}\right)^{\sharp} / \mathscr{E}\left(\mathfrak{a} M_{\xi}\right)$ is an abelian group with exponent dividing 2 . We have indicated in [3, VI] how to find the cardinality of this quotient group (when 2 is unramified). For nondyadic $\mathfrak{p}$ the group $\mathscr{F}(\mathfrak{a} M) / O(\mathfrak{a} M)$ has either 1 or 2 elements, depending on whether $-I$ is in $\mathscr{E}$. For dyadic $p$ some comments on the group are made in [3, VII]. The quotient group $O\left(\mathfrak{a} M_{\xi}\right) / O\left(\mathfrak{a} M_{\xi}\right)^{\#}$ will now be determined explicitly in two special cases.

EXAMPLE 3.5. Let $M=H \perp N \perp \mathfrak{o} w$ where $q(N) \subseteq \mathfrak{D}$. Let $h=$ 
$[(1+\operatorname{ord} 2) / 2]$ so that

$$
M_{*}=H \perp N \perp \mathfrak{p}^{h} w \quad \text { and } \quad M^{*}=H \perp N \perp \mathfrak{p}^{-h} w .
$$

The $\mathscr{E}$-invariant dual lattices are

$$
M_{\xi}=H \perp N \perp \mathfrak{p}^{-k} w \text { and } \quad M^{\xi}=H \perp N \perp \mathfrak{p}^{k} w
$$

where $-h \leqq k \leqq h$. We show, for $a \subseteq 2 \mathfrak{p}^{k+1+h}$, that

$$
O\left(a M_{\xi}\right)=O\left(a M_{\xi}\right)^{\sharp} \text {. }
$$

Let $\varphi \in O\left(\mathfrak{a} M_{\xi}\right)$. Then $\varphi(w)=\varepsilon w+s$ where $s \in \mathfrak{a p}^{-h}(H \perp N)$. For all $t \in H \perp N$, since $\varphi(t) \equiv t \bmod a M_{\xi}$, it follows that $B(s, t)$ is in $a p^{-k}$ and hence $s \in \mathfrak{a p}^{-k}(H \perp N)$. Now $q(w)=q(\varphi(w))$ gives

$$
\varepsilon \equiv 1 \bmod a^{2} \mathfrak{p}^{-2 k} \text {. }
$$

Consequently, $\varphi(w) \equiv w \bmod a p^{-k} M_{*}$ and $\varphi \in O\left(a M_{\xi}\right)^{\ddagger}$.

ExAmple 3.6. Let $M=H \perp N \perp(\mathrm{o} w+\mathrm{o} z)$ where $q(N) \subseteq \mathrm{o}$ and $\mathfrak{o} w+\mathfrak{D} z=\langle A(1,0)\rangle$. Let $h=[(1+\operatorname{ord} 2) / 2]$ so that

$$
M_{*}=H \perp N \perp\left(\mathfrak{p}^{h} w+\mathfrak{o} z\right) \text { and } M^{*}=H \perp N \perp\left(\mathfrak{o} w+\mathfrak{p}^{-h} z\right) .
$$

Fix $0 \leqq i, j \leqq h$; then

$$
M_{\xi}=H \perp N \perp\left(\mathfrak{p}^{i} w+\mathfrak{p}^{-j} z\right) \text { and } \quad M^{\xi}=H \perp N \perp\left(\mathfrak{p}^{j} w+\mathfrak{p}^{-i} z\right)
$$

are a pair of $\mathscr{E}$-invariant lattices (but not all are of this form). If $a \subseteq 4 \mathfrak{p}$

$$
O\left(a M_{\xi}\right) / O\left(a M_{\xi}\right)^{\sharp} \cong\left\{\begin{array}{lll}
\mathcal{D} / \mathfrak{p}^{i+j} & \text { if } & i+j \leqq h \\
\mathcal{O} / \mathfrak{p}^{2 h-i-j} & \text { if } & i+j \geqq h
\end{array} .\right.
$$

We omit the computational details of the proof except to mention that representatives of the various cosets can be obtained from the isometry defined by

$$
\psi_{\varepsilon}(w)=\varepsilon^{-1} w+\frac{1}{2}\left(\varepsilon-\varepsilon^{-1}\right) z, \psi_{\varepsilon}(z)=\varepsilon z
$$

by suitably choosing the unit $\varepsilon$.

\section{REFERENCES}

1. D. G. James, Orthogonal groups of dyadic unimodular quadratic forms, Math. Ann., 201 (1973), 65-74.

2. - Orthogonal groups of dyadic unimodular quadratic forms II, to appear. 3. The structure of orthogonal groups of 2-adic unimodular quadratic forms, J. Number Theory, 5 (1973), 444-455. 
4. 0. T. O'Meara, Introduction to Quadratic Forms, Springer, Berlin-GöttingenHeidelberg, 1963.

Received August 25, 1973. This research was partially supported by the National Science Foundation.

The Pennsylvania State University 


\section{PACIFIC JOURNAL OF MATHEMATICS}

EDITORS

RICHARD ARENS (Managing Editor)

University of California

Los Angeles, California 90024

R. A. Beaumont

University of Washington

Seattle, Washington 98105

J. DugundJI

Department of Mathematics

University of Southern California

Los Angeles, California 90007

D. Gilbarg and J. Milgram

Stanford University

Stanford, California 94305

\section{ASSOCIATE EDITORS}

E. F. BECKENBACH

B. H. NeumanN

F. WOLF

K. YOSHIDA

\section{SUPPORTING INSTITUTIONS}

UNIVERSITY OF BRITISH COLUMBIA CALIFORNIA INSTITUTE OF TECHNOLOGY

UNIVERSITY OF CALIFORNIA

MONTANA STATE UNIVERSITY

UNIVERSITY OF NEVADA

NEW MEXICO STATE UNIVERSITY

OREGON STATE UNIVERSITY

UNIVERSITY OF OREGON

OSAKA UNIVERSITY

\author{
UNIVERSITY OF SOUTHERN CALIFORNIA \\ STANFORD UNIVERSITY \\ UNIVERSITY OF TOKYO \\ UNIVERSITY OF UTAH \\ WASHINGTON STATE UNIVERSITY \\ UNIVERSITY OF WASHINGTON \\ $\stackrel{*}{*} \stackrel{*}{*}{ }^{*}{ }^{*}{ }^{2}$ AMERICAN MATHEMATICAL SOCIETY \\ NAVAL WEAPONS CENTER
}




\section{Pacific Journal of Mathematics}

\section{Vol. 52, No. $1 \quad$ January, 1974}

David R. Adams, On the exceptional sets for spaces of potentials ............ 1

Philip Bacon, Axioms for the Čech cohomology of paracompacta ............ 7

Selwyn Ross Caradus, Perturbation theory for generalized Fredholm operators ..... 11

Kuang-Ho Chen, Phragmén-Lindelöf type theorems for a system of nonhomogeneous equations ............................ 17

Frederick Knowles Dashiell, Jr., Isomorphism problems for the Baire classes .......

M. G. Deshpande and V. K. Deshpande, Rings whose proper homomorphic images are right subdirectly irreducible . . . . . . . . . . . . . . . . . . . . . . . . .

Mary Rodriguez Embry, Self adjoint strictly cyclic operator algebras .............

Paul Erdős, On the distribution of numbers of the form $\sigma(n) / n$ and on some related

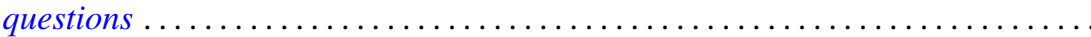

Richard Joseph Fleming and James E. Jamison, Hermitian and adjoint abelian

operators on certain Banach spaces ............................

Stanley P. Gudder and L. Haskins, The center of a poset .................. 85

Richard Howard Herman, Automorphism groups of operator algebras . . . ........

Worthen N. Hunsacker and Somashekhar Amrith Naimpally, Local compactness of families of continuous point-compact relations ....................

Donald Gordon James, On the normal subgroups of integral orthogonal groups ....

Eugene Carlyle Johnsen and Thomas Frederick Storer, Combinatorial structures in

loops. II. Commutative inverse property cyclic neofields of prime-power

order.

Ka-Sing Lau, Extreme operators on Choquet simplexes . . . . . . . . . . . . . . 129

Philip A. Leonard and Kenneth S. Williams, The septic character of 2, 3, 5 and $7 \ldots 143$

Dennis McGavran and Jingyal Pak, On the Nielsen number of a fiber map ........ 149

Stuart Edward Mills, Normed Köthe spaces as intermediate spaces of $L_{1}$ and

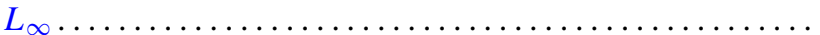

Philip Olin, Free products and elementary equivalence. .

Louis Jackson Ratliff, Jr., Locally quasi-unmixed Noetherian rings and ideals of the principal class.

Seiya Sasao, Homotopy types of spherical fibre spaces over spheres ...

Helga Schirmer, Fixed point sets of polyhedra ...

Kevin James Sharpe, Compatible topologies and continuous irreducible

representations.

Frank Siwiec, On defining a space by a weak base . . . . . . . . . . . . . . . 233

James McLean Sloss, Global reflection for a class of simple closed curves ....... 247

M. V. Subba Rao, On two congruences for primality . .

Raymond D. Terry, Oscillatory properties of a delay differential equation of even

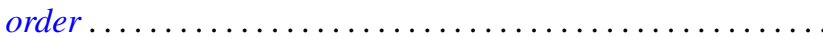

Joseph Dinneen Ward, Chebyshev centers in spaces of continuous functions . .

Robert Breckenridge Warfield, Jr., The uniqueness of elongations of Abelian

groups...

V. M. Warfield, Existence and adjoint theorems for linear stochastic differential

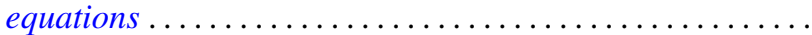

